\title{
Role of exosomes in hepatocellular carcinoma cell mobility alteration
}

\author{
SEN WANG ${ }^{1,2^{*}}$, GENG CHEN $^{1,2^{*}}$, XIAO LIN $^{3}$, XIAOHUA XING $^{1,2}$, \\ ZHIXIONG CAI ${ }^{1,2}$, XIAOLONG LIU $^{1,2}$ and JINGFENG LIU ${ }^{1,2}$
}

\author{
${ }^{1}$ The United Innovation of Mengchao Hepatobiliary Technology Key Laboratory of Fujian Province, \\ Mengchao Hepatobiliary Hospital of Fujian Medical University; ${ }^{2}$ The Liver Center of Fujian Province, \\ Fujian Medical University, Fuzhou, Fujian 350025; ${ }^{3}$ Liver Disease Center, The First Affiliated Hospital of \\ Fujian Medical University, Fuzhou, Fujian 350007, P.R. China
}

Received June 28, 2016; Accepted August 23, 2017

DOI: $10.3892 / \mathrm{ol} .2017 .7257$

\begin{abstract}
Exosomes have gained increased research focus due to their key roles as messengers. The components of exosomes include proteins and RNAs that may be horizontally transferred between adjacent or distant cells. Hepatocellular carcinoma (HCC) is among the most malignant types of cancer worldwide, with exosomes implicated to play a crucial role in its regulation; however, the possible function of exosomes in modulating the motile ability of tumor cells and key molecules in HCC remain largely unknown. To investigate the regulatory effect of exosomes on the motile ability of $\mathrm{HCC}$ cells, exosomes from the culture medium of different HCC origins (high metastatic MHCC $97-\mathrm{H}$ and low metastatic MHCC97-L cells) were isolated for in vitro migration and invasion assays. The results indicated that the motile ability of MHCC97-L cells was significantly increased by pretreatment with MHCC97-H-derived exosomes when compared with MHCC97-L-exosome pretreatment $(\mathrm{P}<0.05)$. To further characterize the function of exosomes at the molecular level, protein profiling of exosomes from different cell origins was performed, which identified 129 proteins. Among these, adenylyl cyclase-associated protein 1 , a protein implicated in HCC metastasis, was significantly enriched in exosomes from cells with high motile ability $(\mathrm{P}<0.05)$. The results of the present study validated the regulatory effect of exosomes on the motile ability of HCC cells. Furthermore, systematic
\end{abstract}

Correspondence to: Professor Xiaolong Liu or Professor Jingfeng Liu, The United Innovation of Mengchao Hepatobiliary Technology Key Laboratory of Fujian Province, Mengchao Hepatobiliary Hospital of Fujian Medical University, 312 Xihong Road, Fuzhou, Fujian 350025, P.R. China

E-mail: xialoong.liu@gmail.com

E-mail: drjingfeng@126.com

\section{${ }^{*}$ Contributed equally}

Key words: exosome, hepatocellular carcinoma, migration and invasion, protein profiling, adenylyl cyclase-associated protein 1 analysis of the protein profiles of exosomes from different origins identified potential factors correlated with HCC metastasis, which may provide a basis for future functional analysis of exosomes regarding their involvement in cancer metastasis and recurrence.

\section{Introduction}

Exosomes are small endosome-derived vesicles that range between 30 and $100 \mathrm{~nm}$ in size, and are actively secreted through the exocytosis pathway (1). The major roles of exosomes are intercellular cross talk and receptor discharge (1-3), and they are typically released from high viability cells, including cancer cells (1). Previous studies have indicated that exosomes are capable of modulating intercellular communication and tumor progression through the transfer of proteins and RNA to adjacent and distant cells $(1,4,5)$. Additionally, the functions of exosomes may vary depending on cell type and intracellular contents (2). In cancer, exosomes are considered to serve essential roles in tumor metastasis by regulating complex interactions between tumor cells and their microenvironment $(6,7)$. However, the regulatory mechanisms of exosomes in tumor metastasis remain to be elucidated. As exosomes are carriers of multiple proteins and RNA molecules, the molecules contained within exosomes may themselves serve key roles in cell-cell communication (1). Thus, proteomics profiling and sequencing are promising platforms for systematically studying exosome components, which may ultimately improve understanding of exosome function.

At present, hepatocellular carcinoma (HCC) is a fatal primary malignancy of hepatocytes (8). Emerging diagnostic tools and novel therapeutic strategies for HCC have substantially improved the clinical outcomes; however, the long-term survival of patients with HCC remains relatively poor due to the high possibility of metastasis and/or recurrence (9). Whether a tumor is likely to undergo local or distant metastasis is principally determined by the metastatic potential of tumor cells and the corresponding microenvironment $(10,11)$. As a major component of the cellular microenvironment, exosomes secreted by different tumor cell types are capable of inducing apoptosis of activated $\mathrm{T}$ cells by promoting the 
expression of cell death ligands (12-14), inhibiting natural killer cell functions $(15,16)$, and promoting the generation of suppressor cells derived from myeloid precursors (13). Additionally, various signaling pathways and genes are involved in the communication between tumor cells and their microenvironment (17). For example, a previous study demonstrated that tumor-activated hepatocytes were capable of altering the expression profiles of colon cancer cells in order to support hepatic metastasis (18). However, despite progress in research regarding the role of exosomes in cell communication, the mechanism by which exosomes alter the metastatic potentials of different cell types, particularly liver cancer cells, still requires further investigation.

To investigate whether exosomes may alter the metastatic potential of cancer cells, the present study used two HCC cell lines with high and low metastatic potential, MHCC97-H and MHCC97-L, for exosome isolation and characterization. To evaluate the regulatory effect of exosomes on the mobility of HCC cells, exosomes from the culture medium of different $\mathrm{HCC}$ origins were isolated for in vitro migration and invasion assays. Additionally, protein profiling was performed on the exosomes from different origins to systematically characterize the content of the exosomes, in order to investigate the regulatory role of exosomes at the molecular level.

\section{Materials and methods}

Cell lines and cell culture. The in-house preserved MHCC97-H and MHCC97-L cell lines were provided by The Second Military Medical University of China (Shanghai, China). All cells were cultured in Dulbecco's modified Eagle medium (DMEM; cat no. C11995500BT; Gibco; Thermo Fisher Scientific, Inc., Waltham, MA, USA), supplemented with 10\% FBS (cat no. 10100-147-FBS; Gibco; Thermo Fisher Scientific, Inc.), $100 \mathrm{U} / \mathrm{ml}$ penicillin and $100 \mu \mathrm{g} / \mathrm{ml}$ streptomycin at $37^{\circ} \mathrm{C}$ in a $5 \% \mathrm{CO}_{2}$ incubator for $24 \mathrm{~h}$.

Exosome purification. Exosomes were isolated using a total exosome isolation kit (cat no. 4478359; Invitrogen; Thermo Fisher Scientific, Inc.) according to the manufacturer's protocol. In brief, $5 \times 10^{6}$ cells were seeded in a volume of $15 \mathrm{ml}$ culture medium at $37^{\circ} \mathrm{C}$ for $24 \mathrm{~h}$, prior to harvesting the cell culture medium. The culture medium was centrifuged at $2,000 \mathrm{x} \mathrm{g}$ at $4^{\circ} \mathrm{C}$ for $30 \mathrm{~min}$ to remove cells and debris. Subsequently, the supernatant containing the cell-free culture medium was transferred to a new tube, and then $15 \mathrm{ml}$ of cell-free culture medium was mixed with $7.5 \mathrm{ml}$ of total exosome isolation reagent. The culture medium/reagent was mixed by vortexing until homogenous, and the samples were incubated at $4^{\circ} \mathrm{C}$ overnight. Following incubation, the samples were centrifuged at $10,000 \mathrm{x}$ for $1 \mathrm{~h}$ at $4^{\circ} \mathrm{C}$. The supernatant was aspirated and discarded, and the pelleted exosomes were resuspended in 1X phosphate-buffered saline (PBS). The exosomes were then washed with $1 \mathrm{X}$ PBS, ultra-filtrated with a molecular weight cut-off (MWCO) of 100,000 Da, and finally dissolved in 1X PBS.

Transmission electron microscopy (TEM). Exosomes isolated from MHCC97-H and MHCC97-L cells were identified for morphology by transmission electron microscopy (TEM) as previously described (19). In brief, exosomes were transferred to a copper grid coated with $0.125 \%$ Formvar in chloroform immediately after isolation. Then the grids were stained with $1 \%(\mathrm{v} / \mathrm{v})$ uranyl acetate in double-distilled water right before examination. A Hitachi 7100 transmission electron microscope was applied for imaging.

Evaluation of HCC cell motile ability following exosome incubation. MHCC97-H and MHCC97-L cells were freshly cultured in DMEM supplemented with $10 \%$ FBS and incubated with $5 \% \mathrm{CO}_{2}$ in air at $37^{\circ} \mathrm{C}$ for $24 \mathrm{~h}$. Exosomes were isolated from high metastatic MHCC 97-H and low metastatic MHCC97-L cells as described above. A total of $10 \mu \mathrm{g}$ pelleted exosomes from each of the MHCC97-H and MHCC97-L cell lines were individually resuspended in $1 \mathrm{ml}$ culture medium. The MHCC97-L cells were mixed with the MHCC97-H- or MHCC97-L-derived exosomes, and the cells were cultured with $5 \% \mathrm{CO}_{2}$ in air at $37^{\circ} \mathrm{C}$ for $6 \mathrm{~h}$ prior to migration and invasion assays.

Migration and invasion assay. Cell migration was evaluated with a Transwell migration assay, while the invasion assays were performed using the Transwell units (Corning Incorporated, Corning, NY, USA) coated with Matrigel (BD Biosciences, Franklin Lakes, NJ, USA), according to the manufacturer's protocols. A total of $1 \times 10^{5} \mathrm{MHCC} 97-\mathrm{H}$ and MHCC97-L cells, and MHCC97-L cells pretreated with exosomes, were seeded onto the upper chamber of the insert in serum-free DMEM (cat no. C11995500BT; Gibco; Thermo Fisher Scientific, Inc.). After $6 \mathrm{~h}$ of incubation at $37^{\circ} \mathrm{C}$, the membrane of the insert was fixed in $100 \%$ methanol at room temperature for $20 \mathrm{~min}$ and stained with crystal violet at room temperature for $20 \mathrm{~min}$. After washing twice with PBS, tumor cells on the upper surface of the filters were removed by wiping with cotton swabs. The number of migrated or invaded cells that had passed through the filter to the lower surface were counted under an inverted microscope (Axiovert A1; Zeiss GmbH, Jena, Germany) in $\sim 30$ fields of view at x200 magnification. Mean values were determined from three independent experiments run in duplicate.

Statistical analysis of in vitro data. Data are presented as the mean \pm standard deviation. Student's $t$ test and analysis of variance (ANOVA) were used to determine whether the MHCC97-L and MHCC94-H groups were statistically significantly different in migratory and invasive ability. Following ANOVA results, Dunnett's test was used as a post hoc test. All data were analyzed with GraphPad Prism 6 (GraphPad Software, Inc., La Jolla, CA, USA) and $\mathrm{P}<0.05$ was considered to indicate a statistically significant difference.

Protein preparation and isobaric tags for relative and absolute quantitation (iTRAQ). For each sample, proteins were precipitated by ice-cold acetone, and subsequently centrifuged at $10,000 \mathrm{xg}$ at $4^{\circ} \mathrm{C}$ for $15 \mathrm{~min}$. A total of $200 \mu \mathrm{l}$ lysis buffer containing $8 \mathrm{M}$ urea, $2 \% \mathrm{SDS}$ and $1 \mathrm{X}$ protease inhibitor cocktail (Roche Diagnostics, Basel, Switzerland) was added to resuspend the precipitate. The protein concentration of the samples was determined with a bicinchoninic acid assay (Beijing Transgen Biotech Co., Ltd., Beijing, 
China) following the manufacturer's protocol. A total of $5 \mu 1$ DTT $(200 \mathrm{mM})$ was then added to the protein samples, and the samples were incubated at $55^{\circ} \mathrm{C}$ for $1 \mathrm{~h}$, after which $10 \mu$ iodoacetamide $(500 \mathrm{mM})$ was added to each sample for $30 \mathrm{~min}$ in the dark at room temperature to alkylate the proteins.

For each sample, the proteins were ultra-filtrated (MWCO, $10,000 \mathrm{Da})$ and dissolved in $100 \mu \mathrm{l}$ triethylammonium bicarbonate $(100 \mathrm{mM})$. The proteins were then digested with sequence-grade modified trypsin (Promega Corporation, Madison, WI, USA), and the resultant peptide mixture was labeled using an iTRAQ reagent kit (Shanghai AB SCIEX, Analytical Instrument Trading Co., Shanghai, China). The peptides were labeled with iTRAQ 8-plex reagent as follows: MHCC97-H and MHCC97-L were labeled with 113 and 114 isobaric tags, respectively; while the peptides from biological repetitions were labeled with 115 and 117, respectively (MHCC97-H), or 116 and 118, respectively (MHCC97-L). Equal amounts of labeled samples $(100 \mu \mathrm{g})$ were then desalted with the Sep-Pak Vac C18 cartridges and dried in a vacuum centrifuge at $4^{\circ} \mathrm{C}$ for $2 \mathrm{~h}$.

High pH reverse-phase separation. A total of $400 \mu \mathrm{g}$ peptide mixture was dissolved in solution A (5\% acetonitrile and $0.1 \%$ formic acid in water; $\mathrm{pH}$ adjusted to 10.0 with ammonium hydroxide), and then fractionated by high $\mathrm{pH}$ separation using an Agilent 1260 Infinity System (Agilent Technologies $\mathrm{GmbH}$, Waldbronn, Germany) connected to a reverse phase column (Durashell C18, $5 \mu \mathrm{m}, 4.6 \times 250 \mathrm{~mm}$; Bonna-Agela Technologies, Inc., Tianjin, China). High pH separation was performed using a linear gradient of solution $\mathrm{B}[0.1 \%$ formic acid in $90 \%$ acetonitrile (ACN); pH adjusted to 10.0 with ammonium hydroxide] from 2 to $40 \%$ over $60 \mathrm{~min}$. The column flow rate was maintained at $700 \mu \mathrm{l} / \mathrm{min}$ and the column temperature was maintained at $45^{\circ} \mathrm{C}$. Following separation, the column was re-equilibrated at the initial conditions for $15 \mathrm{~min}$. A total of 40 fractions were collected, and any two fractions with the same time interval (including, 1 and 21, 2 and 22) were pooled to reduce the fraction numbers. In total, 20 fractions were obtained and dried in a vacuum concentrator at $4^{\circ} \mathrm{C}$ for $2 \mathrm{~h}$.

Low pH nano-liquid chromatography-mass spectrometry (nano-LC-MS)/MS analysis. The fractions were resuspended with $80 \mu \mathrm{l}$ solution $\mathrm{C}(0.1 \%$ formic acid in water $)$, separated by nano-LC and analyzed by electrospray tandem mass spectrometry. The experiments were performed on a Nano LC1000 system (Thermo Fisher Scientific, Inc.) connected to a quadrupole-Orbitrap mass spectrometer (Q-Exactive Plus; Thermo Fisher Scientific, Inc.), equipped with an online nano-electrospray ion source. A total of $2 \mu$ peptide sample was loaded onto the trap column (Thermo Fisher Scientific Inc., Acclaim PepMap C18, $100 \mu \mathrm{m}$ x $2 \mathrm{~cm}$ ) with a flow rate of $10 \mu \mathrm{l} / \mathrm{min}$, and subsequently separated on the analytical column (Acclaim PepMap C18, $75 \mu \mathrm{m}$ x $15 \mathrm{~cm}$; Thermo Fisher Scientific, Inc.), with a linear gradient of solution $\mathrm{D}(0.1 \%$ formic acid in ACN) between 3 and $35 \%$. The column flow rate was maintained at $300 \mathrm{nl} / \mathrm{min}$, the column temperature was maintained at $40^{\circ} \mathrm{C}$, the nebulizer pressure of $\sim 15 \mathrm{MPa}$ and an electrospray voltage of $2.8 \mathrm{kV}$ at the inlet of the mass spectrometer was used. Following the nano-LC separation, the column was re-equilibrated at the initial conditions for $15 \mathrm{~min}$.

The Q-Exactive Plus mass spectrometer was operated in the data-dependent mode to switch automatically between MS and MS/MS acquisition. Survey full-scan MS spectra $(\mathrm{m} / \mathrm{z}, 300-1,500)$ were acquired with a mass resolution of $70 \mathrm{~K}$, followed by 10 sequential high-energy collisional dissociation MS/MS scans with a resolution of $17.5 \mathrm{~K}$. In all cases, one microscan was recorded using a dynamic exclusion of $30 \mathrm{sec}$.

Mass spectrometry data analysis. The raw files from the Q-Exactive instrument were searched against the human database provided by the Universal Protein Resource (http://www.uniprot.org/uniprot, released on 10 April 2014, with 20,264 entries) using Proteome Discoverer (PD) 1.4 (Thermo Fisher Scientific, Inc.). The enzyme specificity of trypsin and a maximum of two missed cleavages were selected for protease digestion. PD was used with a parent ion tolerance of 10 parts per million and a fragment ion mass tolerance of 0.05 Da. Carbamidomethylation of cysteine, as well as iTRAQ modification of the peptide $\mathrm{N}$-terminus and lysine residues, were set as a fixed modification; oxidation of methionine and iTRAQ 8-plex labeling of tyrosine were specified as variable modifications.

A decoy database search strategy was adopted to estimate the false discovery rate (FDR) for peptide identification. Scaffold (version 4.3.2, Proteome Software, Inc., Portland, OR, USA) was used to validate the MS/MS based peptide and protein identifications. The proteins were assembled using the parsimony method and accepted if the peptide FDR was $<1 \%$ and the protein probability was $>99.0 \%$. Proteins containing similar peptides that could not be distinguished based on $\mathrm{MS} / \mathrm{MS}$ analysis alone were grouped to satisfy the principles of parsimony.

Differentially expressed protein filtering and gene ontology $(G O)$ and kyoto encyclopedia of genes and genomes (KEGG) pathway analyses. Proteins with expression fold change $>2$ and Student's t-tests, $\mathrm{P}<0.05$ were filtered as differentially expressed proteins between exosomes isolated from MHCC97-H and MHCC97-L cells. GO and KEGG pathway analyses were conducted using the R packages GO.db (version 3.4.1), KEGG.db (version 3.23) and KEGGREST (version 1.16.1). The P-value threshold was set at 0.01 to filter significantly enriched biological processes and KEGG pathways.

Identification of significantly altered subnetworks. Subnetwork identification was conducted with a heat-diffusion model based on the HotNet 2 algorithm. The expression profile change was used as a heat signal input. The networks used for this analysis were obtained from the Human Protein Reference Database (HPRD) (20), iRefIndex (21) and Multinet (22) as recommended by the algorithm authors. A subnetwork identification result with $\mathrm{P}<0.05$ and minimum edge weight threshold $\delta \geq 0.0003$ were selected for subsequent consensus subnetwork construction. The consensus subnetworks were derived by the following steps: Initially, a complete weighted graph combining all the subnetworks identified from each interaction network was generated. In the weighted graph, 
proteins served as vertices and the edge between any pair of proteins were weighted by the number of networks, in which HotNet 2 reports them in the same subnetwork. Then, the consensus subnetworks were identified by i) initializing the consensus subnetworks with connected components with edge weights $\geq 2$ (connected components were defined as core genes of the consensus subnetworks), and ii) extending the subnetworks by adding similar genes to a given subnetwork until all weight one edges ended in the consensus subnetworks. The construction of the consensus networks was performed using customized Python scripts (version 2.7.13, Python Software Foundation, Wilmington, DE, USA).

\section{Results}

Electron microscopy of isolated exosomes. Exosomes were isolated from the culture media of MHCC97-H and MHCC97-L cells using a total exosome isolation kit. To verify that the isolated structures were exosomes, the isolates were examined by electron microscopy (Fig. 1). The electron images depicted rounded structures with a size range of 50-100 nm in diameter and a cup-shaped morphology, which confirmed the successful isolation of exosomes according to previously described exosome characteristics $(5,23,24)$.

Exosomes from different origins significantly alter the migratory and invasive abilities of cancer cells. The HCC cell lines, MHCC97-H and MHCC97-L, were used in the present study to assess the potential tumor regulatory role of exosomes. MHCC97-H cells exhibited a higher motile ability compared with that of MHCC97-L cells when analyzed by in vitro migration and invasion assays (Fig. 2A). Statistical analyses confirmed that, the migratory and invasive capacity of MHCC97-H cells was $~ 1.5$ times higher than that of the MHCC97-L cells (Fig. 2B; $\mathrm{P}<0.0001$ and $\mathrm{P}<0.005$ ), respectively, which is in accordance with a previous study (25).

To investigate the impact of exosomes on the motile ability of HCC cells, exosomes were individually isolated from MHCC97-H and MHCC97-L cells and incubated with MHCC97-L cells for $6 \mathrm{~h}$. Following incubation, further migration and invasion assays were performed to detect changes in the motile abilities of the MHCC97-L cell groups. As depicted in Fig. 3, according to the migration and invasion assays, MHCC97-L cells incubated with MHCC97-H-derived exosomes exhibited higher motile ability compared with that of MHCC97-L cells incubated with MHCC97-L-derived exosomes. MHCC97-L cells incubated with MHCC97-H-derived exosomes demonstrated significantly increased migratory ability $(\sim 2$-fold; $\mathrm{P}<0.05)$ than those incubated with MHCC97-L-derived exosomes. For the invasion assay, MHCC97L cells incubated with MHCC97-H-derived exosomes displayed higher invasive ability compared with those incubated with MHCC97-L-derived exosomes $(\sim 1.5$-fold; $\mathrm{P}<0.05)$. Taken together, these results indicate the ability of exosomes to regulate the motile capacity of HCC cell lines. It is established that exosomes serve an important role in cell-cell communication by transferring molecules between cells (5). Furthermore, a number of proteins and RNAs have been reported to be enriched in exosomes, which may be responsible for the regulatory role of exosomes $(1,26)$.

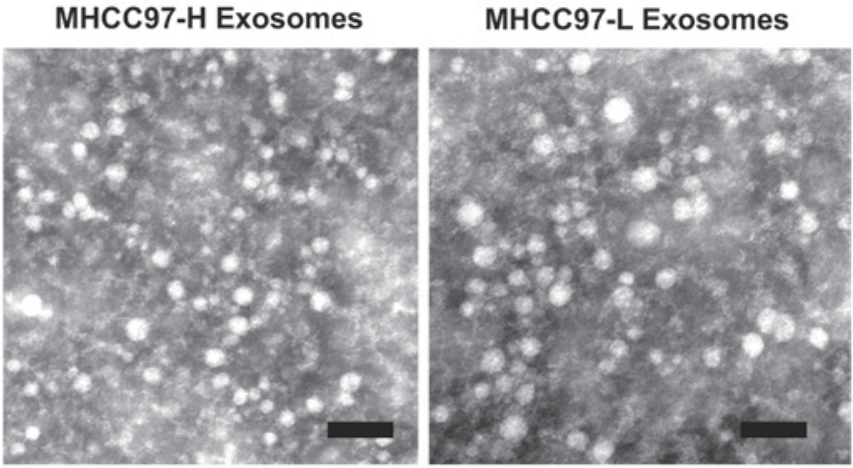

Figure 1. Transmission electron microscopy images of exosomes released from MHCC97-H and MHCC97-L cells. Scale bar, $100 \mathrm{~nm}$.

Therefore, the change in motile ability induced by exosomes in the present study was the basis for subsequent analysis of the molecular content of exosomes.

Mass spectrum analysis of exosomal proteins from different origins. Using a quantitative MS-based discovery strategy, the overall proteomes of exosomes extracted from MHCC97-H and MHCC97-L cells were investigated to characterize the molecular mechanism by which exosomes regulate the motile ability of cells. Exosomes were extracted from the supernatant of the HCC cell lines, and eluted proteins were digested, separated by high $\mathrm{pH}$ reversed-phase LC and analyzed by iTRAQ 2D LC-MS/MS. In total, three biological repeats from MHCC97-H and MHCC97-L exosomes were labeled and processed for quantitative analysis. The representative MS/MS result is depicted in Fig. 4. Reporter ion intensities of representative peptides derived from MHCC97-H and MHCC97-L exosomes are depicted in the inset. Peptides from the MHCC97-H exosomes were labeled with 113, 115 and 117 isobaric reagents, respectively, while peptides from the MHCC97-L exosomes were labeled with 114, 116 and 118 isobaric reagents, respectively. The Scaffold software quantified a total of 129 proteins in the exosome samples isolated from MHCC97-H and MHCC97-L cells.

GO and KEGG enrichment analyses of differentially expressed exosomal proteins in MHCC97-H and MHCC97-L cells. Among the identified genes, adenylyl cyclase-associated protein 1 (CAP1) had a significantly altered expression pattern in exosomes isolated from MHCC97-H cells compared with those from MHCC97-L cells (fold change $\geq 2$; $\mathrm{P} \leq 0.05$ ). Other differentially expressed genes in the MHCC97-H-derived exosomes included peptidylprolyl isomerase A (PPIA), keratin, type I cytoskeletal 20 (KRT20) and inter- $\alpha$-trypsin inhibitor heavy chain family member 4 (ITIH4; fold-change $\geq 1.5$; $\mathrm{P} \leq 0.05$ ). The differentially expressed genes information is presented in Table I. As the aim was to elucidate the roles of exosomes in different biological processes and pathways, GO and KEGG enrichment analyses were performed for all the proteins identified by MS-based discovery. The 129 identified proteins were over-represented in 316 biological processes and 6 KEGG pathways. The top 10 filtered biological processes were 'response to stimulus' (GO:0050896), 'response to stress' (GO:0006950), 'wound healing' (GO:0042060), 'response to 
A
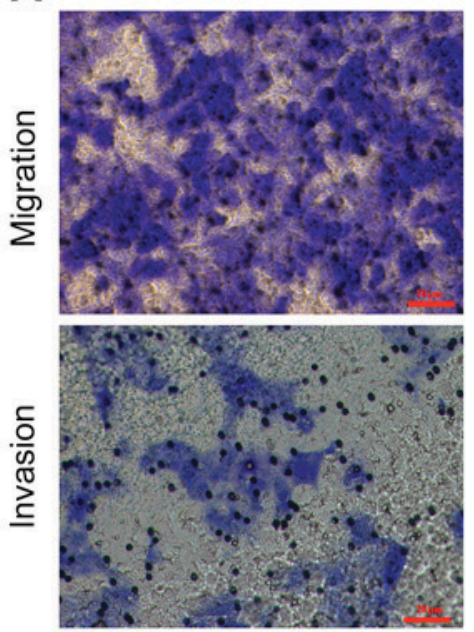

MHCC97-L
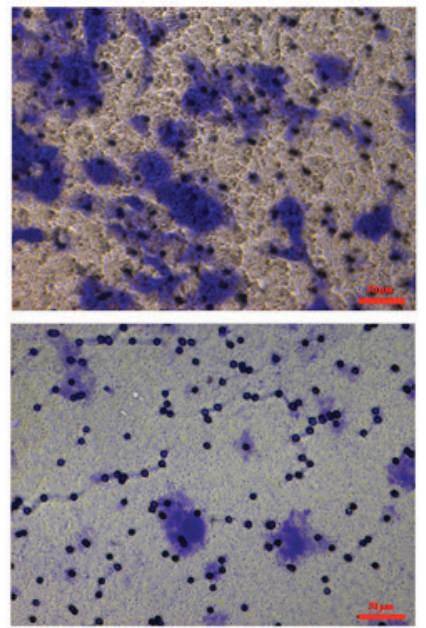

B

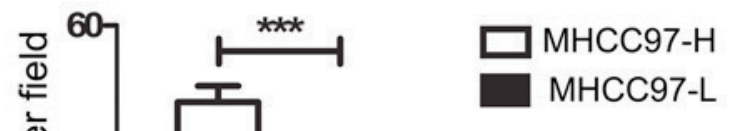

MHCC97-L

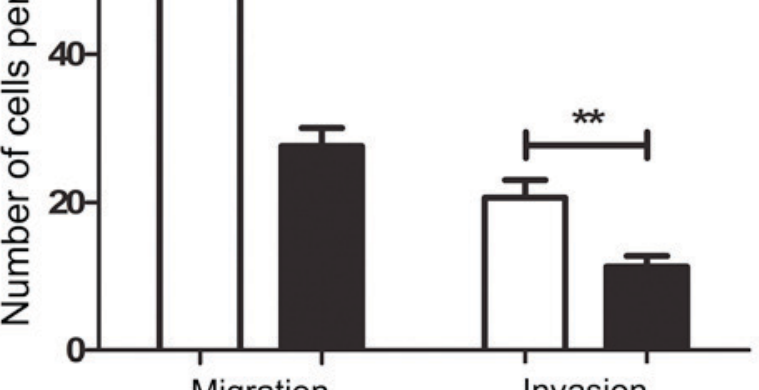

Migration

Invasion

Figure 2. In vitro migration and invasion assays of MHCC97-H and MHCC97-L hepatocellular carcinoma cells. (A) Representative images and (B) quantification of migration and invasion assays, at magnification, $\mathrm{x} 200$; scale bar, $50 \mu \mathrm{m} .{ }^{* * *} \mathrm{P}<0.01,{ }^{* * * *} \mathrm{P}<0.001$.

A MHCC97-H Exosome

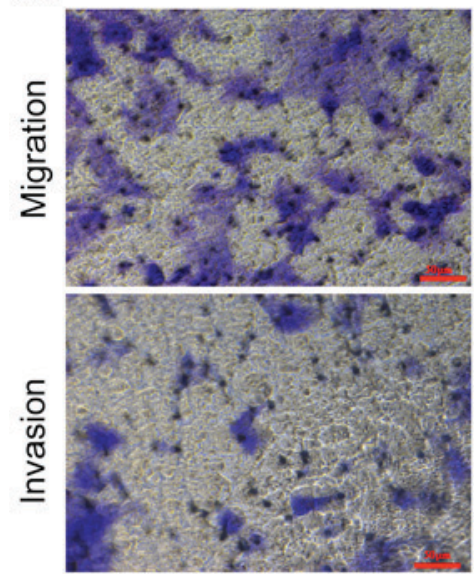

MHCC97-L Exosome
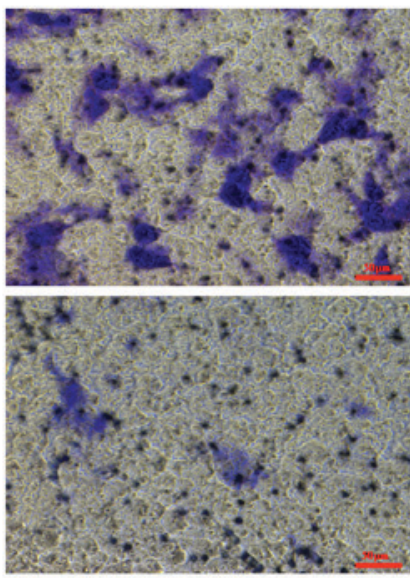

B

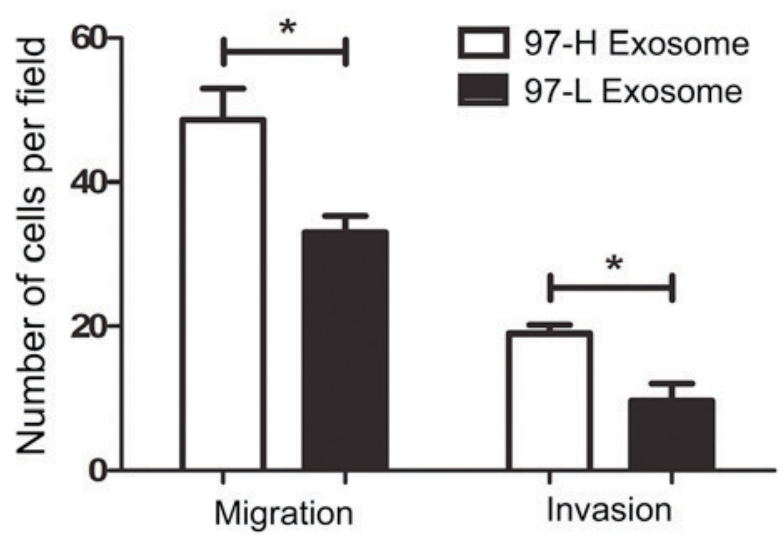

MHCC97-L

Figure 3. Impacts of exosomes from different origins. In vitro migration and invasion assays were performed to assess the altered motile ability of MHCC97-L cells following incubation with exosomes of different origins. (A) Representative images and (B) quantification of migration and invasion assays, at magnification, $\mathrm{x} 200$; scale bar, $50 \mu \mathrm{m} .{ }^{*} \mathrm{P}<0.05$.

wounding' (GO:0009611), 'regulation of body fluid levels' (GO:0050878), ‘blood coagulation' (GO:0007596), ‘coagulation’ (GO:0050817), ‘hemostasis’ (GO:0007599), 'platelet activation' (GO:0030168) and 'platelet degranulation' (GO:0002576), as depicted in Fig. 5. Significantly enriched KEGG pathways were glycolysis/gluconeogenesis, focal adhesion, extracellular matrix-receptor interaction, and complement and coagulation cascades (Table II; $\mathrm{P}<0.001$ ). These results indicated enrichments in proteins involved in cell microenvironment construction and cell responses to environmental stress, thus suggesting the regulatory role of exosomes within the cell microenvironment.

Subnetwork identification based on a heat-diffusion-like model. To elucidate the potential influences of differentially expressed proteins, a heat-diffusion-like model was constructed based on the HotNet2 algorithm (27). This model used the different expression profiles between exosomes from MHCC97-H and MHCC97-L cells as stimulated directed heat signals radiated from the corresponding protein to its interacting partner. Subsequently, diffusion was evaluated in the genome-scale interaction networks to identify significantly altered gene subnetworks, which revealed a combination of proteins across different pathways and complexes. The three interaction networks were extracted from the HPRD (20), iRefIndex (21) and Multinet (22). A single subnetwork was significantly altered in the exosomes of MHCC97-H cells when compared with MHCC97-L cells $(\mathrm{P}=0.0001)$, as depicted in Fig. 6. This subnetwork contained 38 proteins (Presented in Table III), of which the core components principally belonged to three different groups according to their interactions. The first group contained apolipoprotein $\mathrm{E}$ (APOE), low density lipoprotein receptor-related protein 1 (LRP1), talin 1 (TLN1), filamin A (FLNA), lamin A, $\beta$-actin 


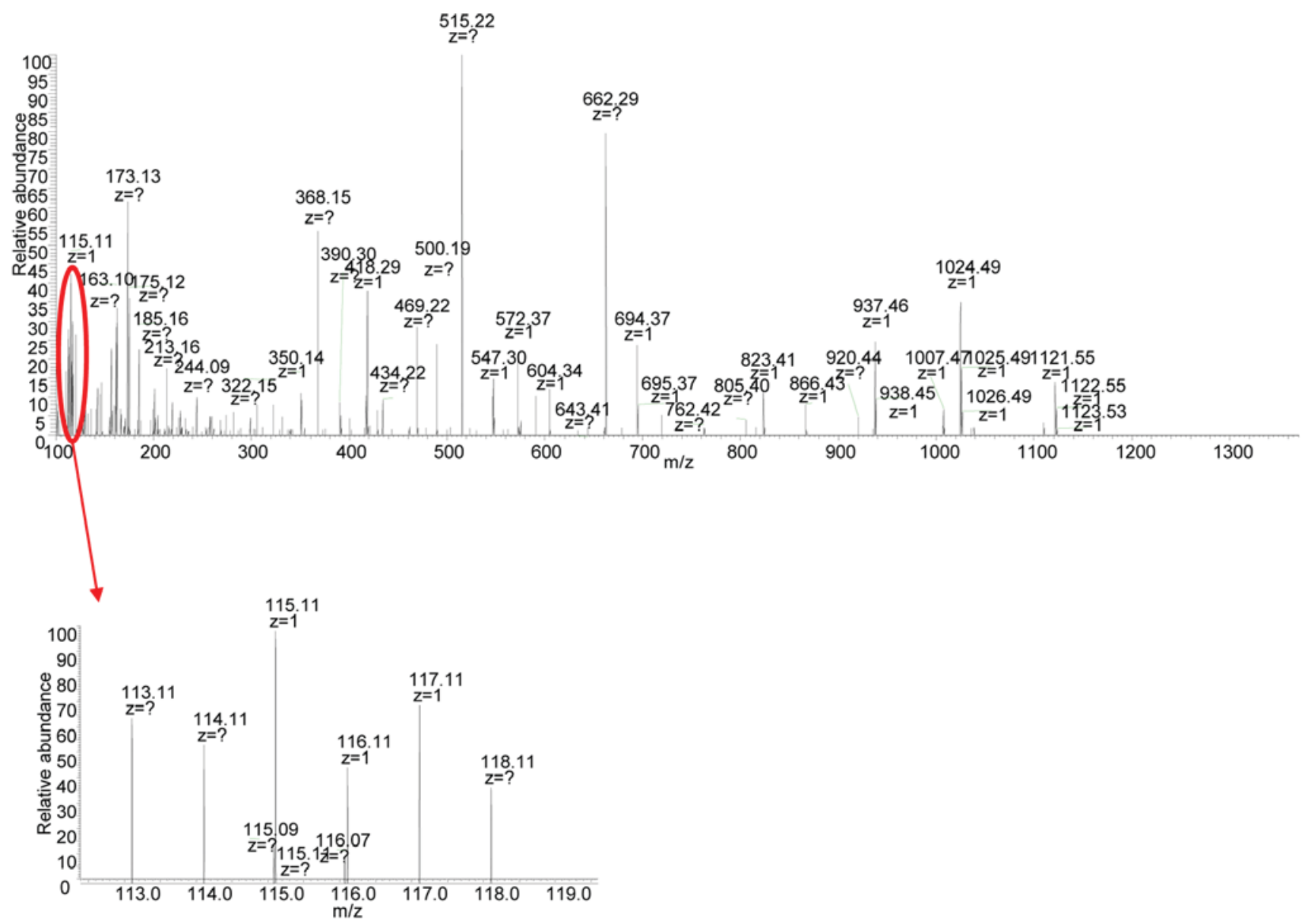

Figure 4. Representative tandem mass spectrometry spectrum of differentially expressed proteins. The inset depicts the isobaric tags for relative and absolute quantitation reporter ion intensities for representative peptides in exosomes from MHCC97-H and MHCC97-L cells. MHCC97-H exosome peptides were labeled with 113, 115 and 117 isobaric tags and MHCC97-L exosome peptides were labeled with 114, 116 and 118 isobaric tags.

(ACTB) and CAP1. These core components were indicated to directly interact and were thus associated. Furthermore, it has been reported that these proteins are associated with cell-cell interactions in cell migration indicating their relevance to cancer metastasis (28). Proteins with close interactions to the core components of the subnetwork (PYGL, G6PD, LUM and TKT) were associated with glucose metabolization, which likely provides the energy required for cell transformation and migration. CAP1, the expression of which was significantly altered between MHCC97-H and MHCC97-L exosomes, was a core component and placed centrally in the subnetwork, implicating it as a key linker gene. The other two highly interactive groups contained laminin subunit $\gamma 1$, nidogen 1 and fibulin 1 (FBLN1), and actinin $\alpha 1$ (ACTN1) and glycogen phosphorylase $\mathrm{B}$, respectively.

All the core components were classified based on function, as the majority were either associated with lipoprotein activity (APOB, APOE and LRP1, depicted as blue nodes in Fig. 6), or with the cell microenvironment and extracellular matrix formation (including FLNA, TLN1, FBLN1, ACTN1/4 and ACTB, depicted as green nodes in Fig. 6). The other core components that did not belong to an interactive group were MET and ras-related nuclear protein (RAN; depicted as red nodes in Fig. 6), indicating that these genes exert a distinct influence in the subnetwork while remaining indirectly associated with the CAP1-centered core. MET and RAN are established cancer-associated genes, particularly regarding cancer development and migration $(29,30)$. Thus, taken together these results indicated an altered fraction of genes that may serve crucial functions in cancer cell-derived exosomes.

\section{Discussion}

The interactions between cancer cells and their microenvironment are important in tumor development (16). In recent studies on HCC, it has been demonstrated that exosomes secreted by cancer cells serve as messengers in cell-cell communication by conveying molecular information between tumor and adjacent cells $(31,32)$. However, while exosomes are a key component of the cellular microenvironment, whether they regulate the migration and invasion of HCC cells remains unresolved. Additionally, the molecular contents of exosomes derived from HCC cells remain largely unclear, and the regulatory role of exosomes regarding metastatic potential requires further elucidation. The present study has indicated that horizontal transfer of exosomes between cells of different origin may lead to changes in the receiver cell metastatic potential, which thus provides insight into how exosomes may be involved in cancer development. This alteration in 
Table I. Differentially expressed exosomal proteins in MHCC97-H and MHCC97-L cells.

\begin{tabular}{lcc}
\hline Protein name & Fold-change & P-value \\
\hline CAP1 & 4.047908066 & 0.035 \\
PPIA & 1.827357238 & 0.028 \\
KRT20 & 1.583989312 & 0.041 \\
ITIH4 & 1.944871172 & 0.048 \\
\hline
\end{tabular}

Table II. Significantly enriched Kyoto Encyclopedia of Genes and Genomes pathways associated with proteins identified by mass spectrum-based discovery.

\begin{tabular}{llcc}
\hline ID & \multicolumn{1}{c}{ Description } & Count & P-value \\
\hline hsa00010 & Glycolysis/gluconeogenesis & 10 & $4.16 \times 10^{-8}$ \\
hsa04510 & Focal adhesion & 16 & $4.69 \times 10^{-8}$ \\
hsa04512 & ECM $^{a}$-receptor interaction & 11 & $5.38 \times 10^{-8}$ \\
hsa04610 & Complement and coagulation & 10 & $7.51 \times 10^{-8}$ \\
\hline
\end{tabular}

${ }^{a} \mathrm{ECM}$, extracellular matrix.

metastatic ability is unlikely to result from a change in the direct expression of several individual proteins, but rather from changes in complex mechanisms consisting of multiple biological pathways (33).

A number of proteins are enriched in exosomes, including membrane trafficking proteins (Rab proteins and Annexins), adhesion molecules (lactadherin) and signal transduction proteins (protein kinases) $(1,26,34)$. Following validation of the regulatory role of exosomes regarding cell mobility, the present study conducted protein profiling of exosomes from different origins to systematically investigate the differentially expressed proteins within the exosomes. Among the differentially expressed proteins, CAP1 was revealed to be significantly upregulated within exosomes from high metastatic HCC cells. CAP1 has been demonstrated to be significantly overexpressed in human HCCs and correlated with HCC metastasis, and thus was suggested to be a potential independent prognostic factor in patients with liver cancer (35). Loss of CAP1 expression may lead to cell polarity defects and altered distributions of actin filaments and mRNA determinants during development (36). Meanwhile, a previous study demonstrated that CAP1 was overexpressed in pancreatic cancer, and indicated an involvement of CAP1 in aggressive pancreatic cancer cell behavior (37).

The present study identified that CAP1 expression was relatively higher in the exosomes of MHCC97-H cells compared with those of MHCC97-L cells, which indicates a regulatory role of CAP1 in cell mobility alterations associated with secreted exosomes. However, a limitation of the present study is that RNA-sequencing screening was not performed to compare the genetic components within exosomes of different origins. It has previously been indicated that the exchange of genetic materials, including mRNA and microRNA, is an

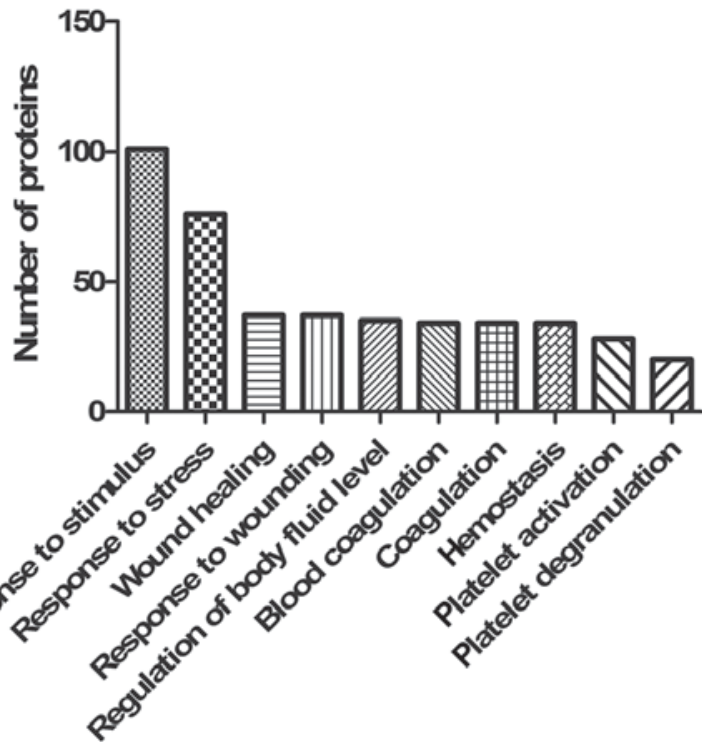

Figure 5. GO analysis of the involved biological processes of the differentially expressed proteins between MHCC97-H and MHCC97-L exosomes. The analysis was performed using the Database for Annotation, Visualization and Integrated Discovery and GO annotations. GO, Gene Ontology.

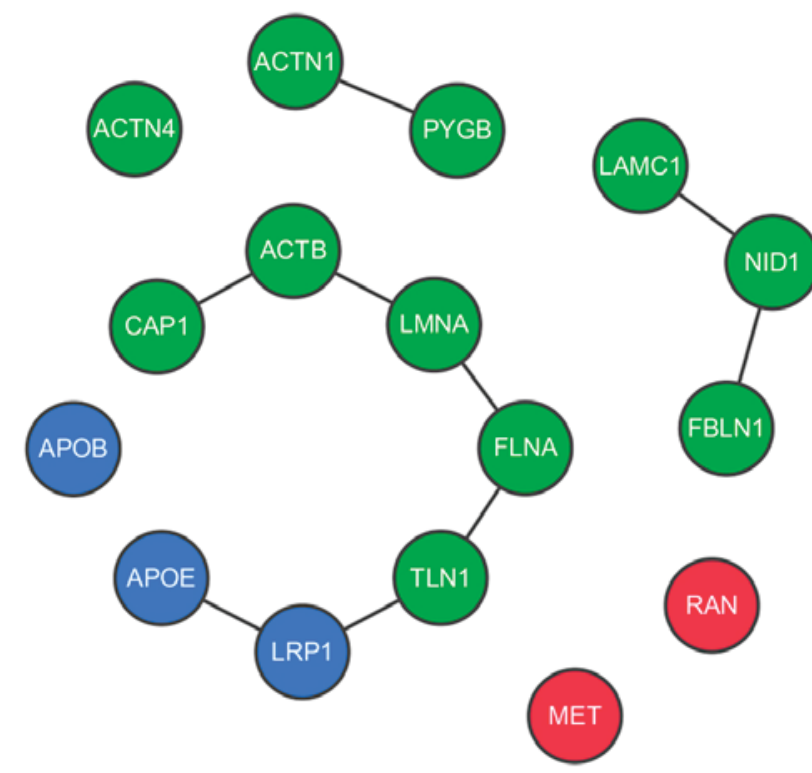

Figure 6. Interactions between core proteins in the subnetwork. Interactions between proteins in the subnetwork determined from three different databases. Each type of protein is depicted as a different color node (blue represents lipoprotein-associated proteins; green represents cell microenvironment and extracellular matrix formation-associated proteins; red represents oncogene encoded proteins). CAP1, adenylyl cyclase-associated protein 1 ; ACTN1/4, actinin $\alpha 1 / 4$; PYGB, glycogen phosphorylase $\mathrm{B}$; ACTB, $\beta$-actin; LMNA, lamin A; FLNA, filamin A; TLN1, talin 1; LRP1, low density lipoprotein receptor-related protein 1; APOB, apolipoprotein B; APOE, apolipoprotein E; LAMC, laminin subunit $\gamma 1$; NID1, nidogen 1 ; FBLN1, fibulin 1; RAN, ras-related nuclear protein.

alternative way of exosome-mediated intercellular communication that may reprogram the recipient cells $(5,38)$. Thus, future studies should focus on the potential regulatory functions of genetic materials within exosomes. Additionally, the level of CAP1 within exosomes and its association with the 
Table III. Consensus subnetworks identified by HotNet2 algorithm.

\begin{tabular}{llr}
\hline Group & \multicolumn{1}{c}{ Component } & Size \\
\hline 1 & APOB, APOE, FLNA, LRP1, PYGL, TLN1, UBA1, FBLN1, LAMC1, NID1, ACTN1, ACTN4, PYGB, & 25 \\
2 & LMNA, ACTB, ANXA2, CAP1, WDR1, G6PD, LUM, PGK1, PPIA, TKT, MET, RAN & 13 \\
\hline
\end{tabular}

regulation of the motile ability of cancer cells requires further characterization.

By constructing a heat-diffusion-like model, a fraction of genes were identified that was significantly altered between exosomes isolated from MHCC97-H and MHCC97-L cells. Genes within this group were divided into several classes. A major part of this subnetwork consisted of lipoprotein genes, which is consistent with the origin of exosomes, as cell-derived vesicles with membranous structure. These lipoproteins are frequently present in different classes of extracellular vesicles, including microvesicles (39), microparticles $(40,41)$ and exosomes $(42,43)$.

Lipoproteins are key components of exosome structure and participate in exosome synthesis, transport and interactions with cells. It has also been reported that APOE and APOB were associated with hepatocyte-derived exosomes (44), while other proteins of this group (including CLIC1 and NRP2) have been associated with the cell microenvironment and extracellular structure $(45,46)$, thus indicating that these proteins may serve key roles in the exosomal regulation of cell metastatic ability. Additionally, it has been reported that tumor cells may modulate the surrounding microenvironment to enhance their metastatic potential through the secretion of exosomes (47). In the present study, the altered expression of exosome lipoproteins may have resulted from differences in the status of the origin cell, as the cell lines possessed different motile abilities. Indeed, several of these proteins (including APOB and APOE) have previously been identified to be associated with cancer $(48,49)$.

FLNA, which crosslinks actin into dynamic extracellular networks and interacts with multiple binding proteins with different biological functions, has been associated with human cancer proliferation, migration and invasion (50), and thus is correlated with cancer development $(51,52)$. Furthermore, TLN1, which encodes a cytoskeletal protein important in the assembly of actin filaments, is considered as a diagnostic and prognostic marker in human HCC $(53,54)$. FBLN1, another component of the fibrillary extracellular matrix, has also been associated with cancer (55). As these cell microenvironment-associated proteins have been associated with cancer migration and metastasis, afforded by their biological functions, alterations in the expression of these proteins in exosomes may impact on the target cell micro environment, and ultimately cause a change in the motile ability of HCC cells, as observed in the present study. Indeed, the results of the present study were consistent with previous observations, indicating that exosomes may not only affect cells but also the surrounding environment (56).

Also noteworthy was the identification of two established oncogenes (MET and RAN) in the differentially expressed subnetwork. The subnetwork structure indicated that these oncogenes were indirectly associated with the extracellular microenvironment and membrane structure, since they are highly interactive with each other (57). This result suggests that oncogenes may affect HCC cell status and migration by changing the extracellular environment, thus leading to a change in the motile ability of cells. MET, which is frequently identified as a key gene in the process of cancer cell migration, serves a role in epithelial-to-mesenchymal transition and contributes to the tumor microenvironment (58). Meanwhile, RAN is associated with the formation and organization of the microtubule network, and previous results have demonstrated that microtubule perturbation may regulate remodeling of the tumor microenvironment to alter cell migratory potential (57). The tumor cell secretome has also been implicated in this mechanism (59). The identification of MET and RAN oncogenes within HCC exosomes indicates that these genes may not only influence the origin cells, but also have an impact on adjacent cells through exosome transportation. Thus, the identification of these genes provides insight into the complex mechanisms of exosome-associated processes and pathways, particularly regarding cancer development and migration. Further studies of these genes may provide more novel perspectives on the association between exosomes and cancer.

In conclusion, the results from the present study indicated that exosomes may alter the metastatic potential of cancer cells. To the best of our knowledge, the present study was the first to conduct protein profiling of exosomes from different HCC cell origins, which identified protein candidates associated with metastasis and recurrence. Collectively, these data may provide the foundation for further studies into the regulatory role of exosomes in cell-cell communication in HCC and other cancers.

\section{Acknowledgements}

The present study was supported by the National Natural Science Foundation of China (grant nos. 31201008 and 31400634), the Specialized Science and Technology Key Project of Fujian Province (grant no. 2013YZ0002-3), the Science and Technology Infrastructure Construction Program of Fujian Province (grant no. 2014Y2005), the Scientific Foundation of Fuzhou City (grant nos. 2015-S-143-1 and 2015-S-143-21), the Natural Science Foundation of Fujian Province (grant no. 2015-J-05173) and the Scientific Research Project of the Health and Family Planning Commission of Fujian province (grant no. 2015-1-96).

\section{References}

1. Thery C, Zitvogel L and Amigorena S: Exosomes: Composition, biogenesis and function. Nat Rev Immunol 2: 569-579, 2002.

2. Stoorvogel W, Kleijmeer MJ, Geuze HJ and Raposo G: The biogenesis and functions of exosomes. Traffic 3: 321-330, 2002. 
3. Valenti R, Huber V, Iero M, Filipazzi P, Parmiani G and Rivoltini L: Tumor-released microvesicles as vehicles of immunosuppression. Cancer Res 67: 2912-2915, 2007.

4. Skog J, Wurdinger T, van Rijn S, Meijer DH, Gainche L, Sena-Esteves M, Curry WT Jr, Carter BS, Krichevsky AM and Breakefield XO: Glioblastoma microvesicles transport RNA and proteins that promote tumour growth and provide diagnostic biomarkers. Nat Cell Biol 10: 1470-1476, 2008.

5. Valadi H, Ekström K, Bossios A, Sjöstrand M, Lee JJ and Lötvall JO: Exosome-mediated transfer of mRNAs and microRNAs is a novel mechanism of genetic exchange between cells. Nat Cell Biol 9: 654-659, 2007.

6. Atay S, Banskota S, Crow J, Sethi G, Rink L and Godwin AK: Oncogenic KIT-containing exosomes increase gastrointestinal stromal tumor cell invasion. Proc Natl Acad Sci USA 111: 711-716, 2014.

7. Luga V, Zhang L, Viloria-Petit AM, Ogunjimi AA, Inanlou MR, Chiu E, Buchanan M, Hosein AN, Basik M and Wrana JL: Exosomes mediate stromal mobilization of autocrine Wnt-PCP signaling in breast cancer cell migration. Cell 151: 1542-1556, 2012.

8. Dimitroulis D, Damaskos C, Valsami S, Davakis $S$, Garmpis N, Spartalis E, Athanasiou A, Moris D, Sakellariou S, Kykalos S, et al: From diagnosis to treatment of hepatocellular carcinoma: An epidemic problem for both developed and developing world. World J Gastroenterol 23: 5282-5294, 2017.

9. Tang ZY, Ye SL, Liu YK, Qin LX, Sun HC, Ye QH, Wang L, Zhou J, Qiu SJ, Li Y, et al: A decade's studies on metastasis of hepatocellular carcinoma. J Cancer Res Clin Oncol 130: 187-196, 2004.

10. Li Y, Tang ZY and Hou JX: Hepatocellular carcinoma: Insight from animal models. Nat Rev Gastroenterol Hepatol 9: 32-43, 2011.

11. He M, Qin H, Poon TC, Sze SC, Ding X, Co NN, Ngai SM, Chan TF and Wong N: Hepatocellular carcinoma-derived exosomes promote motility of immortalized hepatocyte through transfer of oncogenic proteins and RNAs. Carcinogenesis 36: 1008-1018, 2015

12. Andreola G, Rivoltini L, Castelli C, Huber V, Perego P, Deho P, Squarcina P, Accornero P, Lozupone F, Lugini L, et al: Induction of lymphocyte apoptosis by tumor cell secretion of FasL-bearing microvesicles. J Exp Med 195: 1303-1316, 2002.

13. Valenti R, Huber V, Filipazzi P, Pilla L, Sovena G, Villa A, Corbelli A, Fais S, Parmiani G and Rivoltini L: Human tumor-released microvesicles promote the differentiation of myeloid cells with transforming growth factor-beta-mediated suppressive activity on $\mathrm{T}$ lymphocytes. Cancer Res 66: 9290-9298, 2006.

14. Kim JW, Wieckowski E, Taylor DD, Reichert TE, Watkins S and Whiteside TL: Fas ligand-positive membranous vesicles isolated from sera of patients with oral cancer induce apoptosis of activated T lymphocytes. Clin Cancer Res 11: 1010-1020, 2005.

15. Liu C, Yu S, Zinn K, Wang J, Zhang L, Jia Y, Kappes JC, Barnes S, Kimberly RP, Grizzle WE and Zhang HG: Murine mammary carcinoma exosomes promote tumor growth by suppression of NK cell function. J Immunol 176: 1375-1385, 2006.

16. Clayton A, Mitchell JP, Court J, Linnane S, Mason MD and Tabi Z: Human tumor-derived exosomes down-modulate NKG2D expression. J Immunol 180: 7249-7258, 2008.

17. Wu SD, Ma YS, Fang Y, Liu LL, Fu D and Shen XZ: Role of the microenvironment in hepatocellular carcinoma development and progression. Cancer Treat Rev 38: 218-225, 2012.

18. Vidal-Vanaclocha F: The liver prometastatic reaction of cancer patients: Implications for microenvironment-dependent colon cancer gene regulation. Cancer Microenviron 4: 163-180, 2011.

19. Hong C, Funk S and Whiteside T: Isolation of biologically active exosomes from plasma of patients with cancer. Methods Mol Biol 1633: 257-265, 2017.

20. Keshava Prasad TS, Goel R, Kandasamy K, Keerthikumar S, Kumar S, Mathivanan S, Telikicherla D, Raju R, Shafreen B, Venugopal A, et al: Human Protein Reference Database - 2009 update. Nucleic Acids Res 37: D767-D77, 2009.

21. Khurana E, Fu Y, Chen J and Gerstein M: Interpretation of genomic variants using a unified biological network approach. PLoS Comput Biol 9: e1002886, 2013.

22. Razick S, Magklaras G and Donaldson IM: iRefIndex: A consolidated protein interaction database with provenance. BMC Bioinformatics 9: 405, 2008.

23. Raposo G, Nijman HW, Stoorvogel W, Liejendekker R, Harding CV, Melief CJ and Geuze HJ: B lymphocytes secrete antigen-presenting vesicles. J Exp Med 183: 1161-1172, 1996.
24. Caby MP, Lankar D, Vincendeau-Scherrer C, Raposo G and Bonnerot C: Exosomal-like vesicles are present in human blood plasma. Int Immunol 17: 879-887, 2005.

25. Ding SJ, Li Y, Shao XX, Zhou H, Zeng R, Tang ZY and Xia QC: Proteome analysis of hepatocellular carcinoma cell strains, MHCC97-H and MHCC97-L, with different metastasis potentials. Proteomics 4: 982-994, 2004.

26. Matsuo H, Chevallier J, Mayran N, Le Blanc I, Ferguson C, Fauré J, Blanc NS, Matile S, Dubochet J, Sadoul R, et al: Role of LBPA and Alix in multivesicular liposome formation and endosome organization. Science 303: 531-534, 2004

27. Leiserson MD, Vandin F, Wu HT, Dobson JR, Eldridge JV, Thomas JL, Papoutsaki A, Kim Y, Niu B, McLellan M, et al: Pan-cancer network analysis identifies combinations of rare somatic mutations across pathways and protein complexes. Nat Genet 47: 106-114, 2015.

28. Buschow SI, van Balkom BW, Aalberts M, Heck AJ, Wauben M and Stoorvogel W: MHC class II-associated proteins in B-cell exosomes and potential functional implications for exosome biogenesis. Immunol Cell Biol 88: 851-856, 2010.

29. Santhana Kumar K, Tripolitsioti D, Ma M, Grählert J, Egli KB, Fiaschetti G, Shalaby T, Grotzer MA and Baumgartner M: The Ser/Thr kinase MAP4K4 drives c-Met-induced motility and invasiveness in a cell-based model of $\mathrm{SHH}$ medulloblastoma. Springerplus 4: 19, 2015.

30. Zhang F, Yang Z, Cao M, Xu Y, Li J, Chen X, Gao Z, Xin J, Zhou S, Zhou Z, et al: MiR-203 suppresses tumor growth and invasion and down-regulates MiR-21 expression through repressing Ran in esophageal cancer. Cancer Lett 342: 121-129, 2014.

31. Nakamura K, Sawada K, Kinose Y, Yoshimura A, Toda A, Nakatsuka E, Hashimoto K, Mabuchi S, Morishige KI, Kurachi $\mathrm{H}$, et al: Exosomes promote ovarian cancer cell invasion through transfer of CD44 to peritoneal mesothelial cells. Mol Cancer Res 15: 78-92, 2017.

32. Lemoinne S, Thabut D, Housset C, Moreau R, Valla D, Boulanger CM and Rautou PE: The emerging roles of microvesicles in liver diseases. Nat Rev Gastroenterol Hepatol 11: 350-361, 2014.

33. Choi D, Lee T, Spinelli C, Chennakrishnaiah S, D'Asti E and Rak J: Extracellular vesicle communication pathways as regulatory targets of oncogenic transformation. Semin Cell Dev Biol 67: 11-22, 2017.

34. Théry C, Ostrowski M and Segura E: Membrane vesicles as conveyors of immune responses. Nat Rev Immunol 9: 581-593, 2009.

35. Liu Y, Cui X, Hu B, Lu C, Huang X, Cai J, He S, Lv L, Cong X, Liu G, et al: Upregulated expression of CAP1 is associated with tumor migration and metastasis in hepatocellular carcinoma. Pathol Res Pract 210: 169-175, 2014.

36. Baum B, Li W and Perrimon N: A cyclase-associated protein regulates actin and cell polarity during Drosophila oogenesis and in yeast. Curr Biol 10: 964-973, 2000.

37. Yamazaki K, Takamura M, Masugi Y, Mori T, Du W, Hibi T, Hiraoka N, Ohta T, Ohki M, Hirohashi S and Sakamoto M: Adenylate cyclase-associated protein 1 overexpressed in pancreatic cancers is involved in cancer cell motility. Lab Invest 89: 425-432, 2009.

38. Mincheva-Nilsson L and Baranov V: Cancer exosomes and NKG2D receptor-ligand interactions: Impairing NKG2D-mediated cytotoxicity and anti-tumour immune surveillance. Semin Cancer Biol 28: 24-30, 2014.

39. Choi DS, Park JO, Jang SC, Yoon YJ, Jung JW, Choi DY, Kim JW, Kang JS, Park J, Hwang D, et al: Proteomic analysis of microvesicles derived from human colorectal cancer ascites. Proteomics 11: 2745-2751, 2011.

40. Meckes DG Jr, Gunawardena HP, Dekroon RM, Heaton PR, Edwards RH, Ozgur S, Griffith JD, Damania B and Raab-Traub N: Modulation of B-cell exosome proteins by gamma herpesvirus infection. Proc Natl Acad Sci USA 110: E2925-E2933, 2013.

41. Al Kaabi A, Traupe T, Stutz M, Buchs N and Heller M: Cause or effect of arteriogenesis: Compositional alterations of microparticles from CAD patients undergoing external counterpulsation therapy. PLoS One 7: e46822, 2012.

42. Tauro BJ, Greening DW, Mathias RA, Ji H, Mathivanan S, Scott AM and Simpson RJ: Comparison of ultracentrifugation, density gradient separation, and immunoaffinity capture methods for isolating human colon cancer cell line LIM1863-derived exosomes. Methods 56: 293-304, 2012. 
43. Liang B, Peng P, Chen S, Li L, Zhang M, Cao D, Yang J, Li H, Gui T, Li X and Shen K: Characterization and proteomic analysis of ovarian cancer-derived exosomes. J Proteomics 80: $171-182,2013$

44. Ramakrishnaiah V, Thumann C, Fofana I, Habersetzer F, Pan Q, de Ruiter PE, Willemsen R, Demmers JA, Stalin Raj V, Jenster G, et al: Exosome-mediated transmission of hepatitis $\mathrm{C}$ virus between human hepatoma Huh7.5 cells. Proc Natl Acad Sci USA 110: 13109-13113, 2013.

45. Setti M, Osti D, Richichi C, Ortensi B, Del Bene M, Fornasari L, Beznoussenko G, Mironov A, Rappa G, Cuomo A, et al: Extracellular vesicle-mediated transfer of CLIC1 protein is a novel mechanism for the regulation of glioblastoma growth. Oncotarget 6: 31413-31427, 2015.

46. Landskron G, De la Fuente M, Thuwajit P, Thuwajit $C$ and Hermoso M: Chronic inflammation and cytokines in the tumor microenvironment. J Immunol Res 2014: 149185, 2014.

47. Park JE, Tan HS, Datta A, Lai RC, Zhang H, Meng W, Lim SK and Sze SK: Hypoxic tumor cell modulates its microenvironment to enhance angiogenic and metastatic potential by secretion of proteins and exosomes. Mol Cell Proteomics 9: 1085-1099, 2010.

48. Ahles T, Li Y, McDonald BC, Schwartz GN, Kaufman PA, Tsongalis GJ, Moore JH and Saykin AJ: Longitudinal assessment of cognitive changes associated with adjuvant treatment for breast cancer: The impact of APOE and smoking. Psychooncology 23 $1382-1390,2014$

49. Chandler P, Song Y, Lin J, Zhang S, Sesso HD, Mora S, Giovannucci EL, Rexrode KE, Moorthy MV, Li C, et al: Lipid biomarkers and long-term risk of cancer in the Women's Health Study. Am J Clin Nutr 103: 1397-1407, 2016.

50. Zhang K, Zhu T, Gao D, Zhang Y, Zhao Q, Liu S, Su T, Bernier M and Zhao R: Filamin A expression correlates with proliferation and invasive properties of human metastatic melanoma tumors: Implications for survival in patients. J Cancer Res Clin Oncol 140: 1913-1926, 2014.
51. Tian ZQ, Shi JW, Wang XR, Li Z and Wang GY: New cancer suppressor gene for colorectal adenocarcinoma: Filamin A. World J Gastroenterol 21: 2199-2205, 2015.

52. Wieczorek K and Niewiarowska J: Filamin A as a mediator of alterations in cancer cells. Postepy Biochem 60: 77-83, 2014 (In Polish).

53. Zhang JL, Qian YB, Zhu LX and Xiong QR: Talin1, a valuable marker for diagnosis and prognostic assessment of human hepatocelluar carcinomas. Asian Pac J Cancer Prev 12: 3265-3269, 2011.

54. Youns MM, Abdel Wahab AH, Hassan ZA and Attia MS: Serum talin-1 is a potential novel biomarker for diagnosis of hepatocellular carcinoma in Egyptian patients. Asian Pac J Cancer Prev 14: 3819-3823, 2013

55. Xiao W, Wang J, Li H, Xia D, Yu G, Yao W, Yang Y, Xiao H, Lang B, Ma X, et al: Fibulin-1 is epigenetically down-regulated and related with bladder cancer recurrence. BMC Cancer 14: 677, 2014.

56. Hosseini-Beheshti E, Choi W, Weiswald LB, Kharmate G, Ghaffari M, Roshan-Moniri M, Hassona MD, Chan L, Chin MY, Tai IT, et al: Exosomes confer pro-survival signals to alter the phenotype of prostate cells in their surrounding environment. Oncotarget 7: 14639-14658, 2016.

57. Yuen H, Chan K, Grills C, Murray JT, Platt-Higgins A, Eldin OS, O'Byrne K, Janne P, Fennell DA, Johnston PG, et al: Ran is a potential therapeutic target for cancer cells with molecular changes associated with activation of the PI3K/Akt/mTORC1 and Ras/MEK/ERK pathways. Clin Cancer Res 18: 380-391, 2012.

58. Banyard $\mathrm{J}$ and Bielenberg D: The role of EMT and MET in cancer dissemination. Connect Tissue Res 56: 403-413, 2015

59. Berbari NF, Sharma N, Malarkey EB, Pieczynski JN, Boddu R, Gaertig J, Guay-Woodford L and Yoder BK: Microtubule modifications and stability are altered by cilia perturbation and in cystic kidney disease. Cytoskeleton (Hoboken) 70: 24-31, 2013. 